Andrea Horváth

\title{
(Nicht-)Fiktive Kriegserfahrungen und Kriegsgeschichten
}

\author{
(Non-)fictitious war experiences and war stories
}

\begin{abstract}
The First World War not only destroyed almost all life, it also restructured established traditions and structures and completely redefined social relations. In my contribution I try to present the generation and time experience of that time by examining in more detail the reactions of German intellectuals to the shattering of occidental values. It is about the crisis of the monarchical principle, the call for democratization, the experience of death and the evaluation of knowledge.
\end{abstract}

Keywords: World War I, generation, intellectuals, democratization, interpretations of war

Die Kriegsgeschichte ist nicht die ganze Geschichte, wie man spätestens seit Jacob Burkhardt weiß. Dennoch sind Kriege geschichtliche Ereignisse und schaffen Zeitzäsuren. ${ }^{1}$ Mit dem Ersten Weltkrieg begann 1914 eine Epoche der Gräuel. Der Krieg zerstörte nicht nur fast das ganze Leben, sondern er entwickelte gewachsene Traditionen und Strukturen und definierten gesellschaftliche Verhältnisse ganz neu. In meinem Beitrag versuche ich, die damaligen Generations- und Zeiterfahrung darzustellen, indem die Reaktionen der deutschen Intellektuellen auf die Zertrümmerung der abendländischen Werte detaillierter unter die Lupe genommen werden. Es geht aber dabei um eine Masse der literarischen Weltkriegsproduktion, so kann ich mich in meiner literaturwissenschaftlich-geschichtliche Arbeit nur auf ausgewählte Autorinnen und Autoren und Themen konzentrieren.

Laut Kurt Flasch können auch Strukturen ,inhaltlicher" Natur in der großen Masse von Texten über den Weltkrieg erkannt werden. Es zeigten

1 This paper was supported by the János Bolyai Research Scholarship of the Hungarian Academy of Sciences. Korrekturleser: Karl Katschthaler.

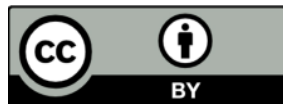


sich bei der geforderten summarischen Betrachtung Konvergenzen in den Resultaten, wiederkehrende Reflexionen zur theoretischen, gesamtgeschichtlichen und ästhetischen Erfahrung des Krieges. Das Nachdenken über seine reale und fortwirkende Bedeutung, oft im Nachhinein ausgesprochen, habe sich innerhalb bestimmter thematischer Vorgaben bewegt. Autoren, die sich fragten, wie die Weltkriegserfahrung die intellektuelle, die kulturelle Situation und damit auch die politische insgesamt nach 1918 verändert habe, hätten aus gemeinsamen Problemen, Lösungsmustern und Metaphern geschöpft, mit denen sie in Deutschland bis 1933, in Österreich und der Schweiz bis etwa 1938/39 gearbeitet hätten. ${ }^{2}$

Wenn man an die intellektuelle Aufarbeitung des Weltkriegs denkt, fragt man noch immer nach den philosophischen, literarischen, künstlerischen und politischen Konsequenzen. Als Erstes zeichnet sich als allgemeine intellektuelle Kriegsfolge eine Krise des monarchischen Prinzips ab. 1918 bedeutete für die meisten das definitive Ende des Gottesgnadentums als politisches und kosmologisches Modell. Das führte zu einer Strukturkrise der Gesellschaft der Mittelmächte, und zwar nicht nur der aristokratischen, sondern auch der bürgerlichen Schichten. Die Krise erreichte besonders stark die evangelische Kirche in Deutschland. Die Monarchie und das Konzept von Ordnung als Unterordnung, diese Tradition von exemplarischer Funktion verschwand zwar nicht völlig als Wertkonzept und Erziehungsideal, aber es fehlte fortan die sichtbare politische Abstützung und die Korrektur durch die Empirie. Das monarchische Prinzip hatte nicht nur die Berliner Politik bestimmt, auch Gymnasialdirektoren und Bahnhofsvorstände hatten es nach Kräften nachgeahmt. Seine Krise setzte Reflexionen über eine Parlamentarisierung frei, führte aber auch zur Suche nach einem neuen Typus höchster Autorität: Der charismatische Führer erschien bei Max Weber und Max Scheler und Carl Schmitt schrieb über die Diktatur. Auch die Mythisierung des Helden antwortete auf diese Situation und endete daher nicht im November 1918.

Zweitens war der Ruf nach „Demokratisierung“ auch eine relevante Folge der Kriegserfahrung. Die Hierarchie des „Standeskonzeptes“ ist in die Krise geraten, die Arbeiter und die Frauen verlangten wenigstens formal gleiche Rechte und so lag die Stufenordnung als Weltmodell in den letzten Zügen. Als dritte Folge brachte der Krieg eine umwälzende Erfahrung des „Zeitbruchs“. Allgemein war das Bewusstsein, dass im Weltkrieg eine „Welt unterging oder schon untergegangen war". ${ }^{3}$ Im Sinne von Bergson wird die Zeiterfahrung durch die Erfahrung von Diskontinuitäten der privaten Le-

2 Flasch 2000: 259-260.

3 Flasch 2000: 260. 
bensführung, der politischen Organisation, der ökonomischen Basis (Geldentwertung) immer problematischer. Viertens brachte der Weltkrieg für das gesellschaftliche Denken einen markanten Verlust des „Vernunftvertrauens“, ein offenbares Scheitern vieler Arten von „Rationalismus“. Durch die Erfahrung der Gleichgültigkeit von Intentionen haben diejenigen auch einen anderen Krieg erwartet, die ihn gewollt haben. Es schien so, als ob der Zufall alles dominierte, die einfache Relation von Meinung und Resultat gab es nicht mehr. Das Verhältnis von innen und außen trat in eine epochal neue, in eine kritische Phase, eine der Enttäuschung über die höchsten Instanzen. Die Kriegsintellektuellen haben nicht mehr an die idealistischen Wendungen des Guten geglaubt, weder an Gott noch an dem Kaiser. ${ }^{4}$

Der millionenfache Tod junger Männer veränderte das Verhältnis von Leben und Tod. Die moralisierenden Politikschriftsteller forderten die Opferbereitschaft bis zum Tode. Die Schützengrabenerfahrung zeigte, dass dieser Tod auf keine moralische Disposition Bezug nahm, dass er bisherigen psychologischen Vorwegnahmen widersprach. Das Missverhältnis von Rhetorik und Todeserfahrung wurde offensichtlich und verstärkte die Tendenz, den Tod vergessen zu wollen. Denker und Dichter kritisierten diese wohlverständliche Tendenz als Ausweichen; sie wollten den Tod gegenwärtig halten. Leopold Ziegler veröffentlichte im Winter 1914/15 seinen Essay Vom Tod, Scheler schrieb über die Einheit von Leben und Tod, Rilke verfasste 1919 sein Requiem.

Eine weitere Folge der Kriegserfahrung war, dass sich die Rolle des Staates veränderte. Sie erhielt im Krieg eine Dominanz, wie sie zuvor kaum denkbar gewesen wäre. Der »Staat» wurde mehr oder weniger ein »totaler Staat«. Diese Erfahrung setzte sich um in die Rede vom Ende des Individualismus, des Subjektivismus und des Liberalismus. Das Zeitalter des Privaten ging zu Ende, die Menschen erfuhren sich als abhängig, als sekundär. Im Krieg und in der Nachkriegszeit kam es zur Erfahrung der Geringfügigkeit der Persönlichkeit in der Geschichte.

Die Kriegserfahrung veränderte die Bewertung des „Wissens“. Die offiziellen Kriegsdeutungen sagten nichts, die akademischen Erklärungen waren leer, die Menschen fühlten sich allein gelassen. Dies führte einerseits zur Einsamkeitserfahrung des zum bloßen Mittel herabgesetzten Individuums, andererseits zur Neueinschätzung des Wissens und Schreibens, insbesondere zur Kritik an der bloß »historischen Gelehrsamkeit«. Immer mehr Direktheit und Lebensbezug wurde verlangt, Traditionen wurden gebrochen, Historismus ist auf einmal passé. Der Staat ist zu neuer Macht gekommen,

4 Mayer 2010: 48-50. 
nicht nur internationale Verträge wurden missachtet, sondern auch Privatmoral und politische Ethik. Man sprach von „ethischem Imperialismus": Der Staat war demnach eine ethische Größe, seine Selbsterhaltung und seine Machtsteigerung eo ipso sittlich - nur nicht nach dem beschränkten Horizont privater Ethik.

Der Krieg tauchte nicht nur die alten Instanzen von Vernunft und Freiheit, von Willen und Geschichte, von Persönlichkeit und Großgruppe in neues Licht, sondern führte auch zur Problematisierung der "Sprache«. Sprache wurde als Kommando, als nationale Rhetorik, als abgehobene Kriegspredigt, als totes Formular und als Propagandalüge neu aktualisiert. Kriegsgedichte und Kriegsreden bestanden aus unüberschaubaren Formen. Dies verstärkte das Bewusstsein vom Sprachzerfall, das Hofmannsthal schon vor dem Krieg im sogenannten Chandos-Brief ausgesprochen hatte, während der Papst der Sprachkritik, Fritz Mauthner, ganz kritiklos in die Weltkriegsrhetorik einstimmte.

Die Intellektuellen haben diese Wandlungen oft mit Verspätung verfolgt. In ihren politischen Haltungen findet man Verschiebungen, man sucht vergeblich nach konkreten Positionierungen. Wie Flasch feststellt, es gibt kein zeitfreies Gesamtbild. Wie es am Beispiel von Thomas Mann gezeigt werden kann, der 1914/15 zu den bedeutendsten Kriegsschriftstellern gehörte, können von dem 1915 erschienenen Buch über Friedrich und die Große Koalition bis zu Von deutscher Republik. (1922) eher Wege gezeichnet werden, wie sich die Tonarten der Autoren verändert haben. ${ }^{5}$

Der Weltkrieg hat die Bewertung von Traditionen verändert, Motive kehren zurück, die früher keine Beachtung gefunden haben. Das gilt für den Expressionismus in der Malerei und in der Lyrik. Es gibt aber zwei noch charakteristischere Beispiele: Franz Kafka und Oswald Spengler. Sie bestimmten in höchster Relevanz die deutsche intellektuelle Nachkriegssituation. Schon seit 1912 gab es Vortexte über Bedrohung und Apokalypsis, negative Vorkriegsgefühle beherrschten die Kriegstexte. Fichte entwickelte sich zum meistzitierten Philosophen, bei Nietzsche suchte man die Rechtfertigung der Rücksichtslosigkeit und Bedenkenlosigkeit, Kierkegaard ist für alle bekannt geworden und anerkannt worden und Georg Lukács entschied sich 1917 für den Marxismus. ${ }^{6}$

Die Weltkriegstexte hatten nicht nur regelmäßige Themenvorgaben und Muster für ihre Ergebnisse, sondern variierten auch eine limitierte Anzahl von Auffassungsweisen. Sie folgten bestimmten Denk- und Redestilen, die

5 Flasch 2000: 265.

6 Koch 2005: 29-30. 
durch die Kriegserfahrung teils bestätigt, teils erzeugt, teils eliminiert und teils verändert worden sind. Mit ein wenig Leseerfahrung lässt sich daher schon anhand eines einzelnen Satzes, erst recht bei einem längeren Text ziemlich sicher bestimmen, ob er vor oder nach dem Weltkrieg geschrieben worden ist. Der Weltkrieg hat nicht nur viele Alltagseinzelheiten verändert, sondern auch Stile des Lebens, des Denkens und des Sprechens. Damit kann nicht gemeint sein, dass es einen einheitlichen Denkduktus für alle vor und einen anderen für alle nach dem Krieg gegeben habe, aber es zeigen sich gedankliche und verbale Knotenbildungen, Wandlungen in den formalen Tendenzen des Auffassens und Schreibens. Die Differenzen etwa zwischen Scheler und Bloch, zwischen Ball und Borchardt können sie nicht vergessen machen, aber je tiefer wir auf den Stufen intellektueller Qualität steigen, um so auffälliger werden die gemeinsamen Denkstrukturen deutscher Weltkriegstexte.

Das gesamte Gelände ist noch unübersichtlich und dazu kommt die schwer zu umgehende Gefahr, sich an die „großen“ Autoren zu halten. Es gibt deutsche Kriegsreden von solcher Banalität und Selbstsicherheit, dass sie heute nur noch lächerlich wirken. Aber auch diese Reden gehören zum Gesamtbild. Niemand hat beispielsweise einen Überblick, was in der Presse an theoretisch Ambitioniertem alles vorgebracht worden ist. ${ }^{7}$

Während des Krieges produzierten die Intellektuellen nicht nur auf theoretischer Ebene, sondern sie haben wirklich versucht, die Stellung einzelner gesellschaftlicher Gruppen nicht nur zu bestimmen, sondern auch zu verbessern. Diese Hilfefunktion der Kriegstexte ist gerade in der Alltagrealität von größter Aktualität, da der Weltkrieg die gesellschaftliche Hierarchie in die Krise gestürzt hat. Die nichtdominanten Gruppen der Gesellschaft, die Frauen, die Juden und die Katholiken, konnten ihren sozialen Status verbessern. Zwischen 1914 und 1919 erhielten Frauen das Wahlrecht und wurden an den Universitäten zugelassen und die Katholiken und die Juden kamen auch in höhere politische Positionen.

Der Anteil von Frauen an nichtfiktiver Weltkriegsliteratur ist von großer Relevanz. Sie nahmen an der intellektuellen Mobilmachung wie an der Demobilisierung teil. Die Schwestern Elisabeth und Mathilde MetzdorfTeschner publizierten ein 40-Seiten-Heft über Die allgemeine Wehrpflicht der Frau während des Krieges (1914). Agnes von Harnack, die Tochter des berühmten Theologen, die auch die Biographie ihres Vaters verfasst hat, hielt einen Vortrag Der Krieg und die Frauen, den sie publiziert hat und der öfters neu gedruckt wurde. Aus der Schweiz meldete sich unter den ersten Friedensstimmen 1916 Clara Ragaz mit Die Frau und der Friede. Rosa Luxemburg

7 Koch 2014: 98. 
bekämpfte seit 1916/17 literarisch den Militarismus und die Halbheiten der Sozialdemokratie, zuerst in der Zeitschrift Die Aktion, seit 1918 in Die Rote Fabne. Nach ihrer Ermordung erschienen die Briefe aus dem Gefängnis.

Aber auch Männer beschäftigten sich mit Frauenfragen: Heinrich Planck formulierte den Text Was lehrt der Krieg unsere Frauen und Töchter? (1916), Pastor Friedrich Niemöller schrieb 1916 über Der deutschen Kriegsfrauen Not und Hilfe, und Friedrich Naumann verfasste einen Artikel über Die Fran im Kriege. ${ }^{8}$

Gertrud Bäumer arbeitete bei der Zeitschrift Die Hilfe, sie war von 1910 bis 1919 Vorsitzende des Bundes deutscher Frauenvereine. Sie veröffentlichte mit Helene Lange zusammen die Zeitschrift Die Frau. Als die wichtigste Vertreterin der deutschen Frauenbewegung hatte Bäumer 1914 die Möglichkeit, in der Reihe Der deutsche Krieg eine politische Flugschrift zu verfassen. Getrud Bäumer knüpft, wie fast alle Autoren im Herbst 1914, an die Rauscherfahrung der Mobilmachung an: „Eine Erschütterung, eine Erhebung, ein Weitwerden der Seelen." "Sie gibt dann der weiblichen Rolle eine nationale und soziale Bestimmung und endet mit dem Aufruf zum Opferwillen: „Die deutschen Frauen werden ihrem Lande mit täglich gefestigter Kraft und täglich geklärter Einsicht durchhalten helfen, und sie werden einst die Schwelle des Friedens reifer, treuer und ernst überschreiten."10

Dies ist in verhaltener Form die übliche Rhetorik über die Werte schaffende Rolle des Krieges, als mache die Kriegserfahrung die deutschen Frauen "treuer" und als seien sie nicht auch ohne den Krieg schon „ernst“ genug gewesen. Bäumer spricht in rigoros-moralistischem Ton von der „unwägbaren Wehrleistung der Frauen“, die im „Erziehungswerk deutscher Mütter” und in der „Fürsorge deutscher Gattinnen“11 bestehe, wendet sich dann aber lebenskundig und organisationserfahren den Problemen der Haushaltsführung und vor allem den sozialen Kriegsaufgaben zu. Ihre Rede zerfällt in zwei ungleich große Teile: Im ersten, kleineren Teil fasst sie die Mobilmachungserfahrung der Frauen zusammen, während der zweite Teil der Sozialpolitik gilt. Für die Suche nach einer weiblichen Sichtweise bei der intellektuellen Arbeit am Weltkrieg kommt nur der erste Teil in Betracht.

In der Einleitung zitiert Gertrud Bäumer einen Brief aus dem Jahr 1813, verfasst von einer Gräfin Sophie Schwerin aus altem preußischen Militäradel. Die Gräfin fragt nach dem Sinn des Todes ihres Mannes im Krieg. Bäumer kritisiert den „leidenschaftlichen Egoismus einer solchen Liebe“ und behauptet, keine Frau, die den August 1914 erlebt habe, könne eine solche

8 Flasch 2000: 291.

9 Bäumer 1914: 30.

10 Bäumer 1914: 30 .

11 Bäumer 1914: 6. 
„beinahe frevelhafte Ausschließlichkeit“ noch nachempfinden, denn die deutschen Frauen hätten jetzt erlebt, dass das Vaterland nicht nur das ihrer Gatten und Söhne sei, sondern dass es „eine lebendige, beseligende und erschütternde Macht in ihrem eigenen Herzen ist" ".12 Sie sähen sich nicht mehr wie 1813 dem Staat gegenüber, sondern nähmen nun jubelnd von ihm ihre weibliche Identität erst entgegen: „Ja, wir Frauen sind in diesen Augustwochen wie in eine neue Welt eingetreten." 13 Auf die Mobilmachung, auf dieses „Einswerden, dies rauschende Zusammenfließen unserer Volkskraft in einen ehernen Willen, die heroische Stimmung unserer Truppen" 14 antworte die weibliche Seele mit einer Mystik der Nation,

„mit heiligem Schaudern. Keine Liebe, so sehr sie uns beseligte oder schmerzte, keine Kunst, so sehr sie uns erhob und hinriß, keine Arbeit und kein Glück haben uns diese Erhebung kennen lehren. In uns sprach, fühlte, wollte Deutschland, unsere persönliche Seele ging auf in der Seele unseres Volkes."15

Es bestand in der Weltkriegsliteratur insgesamt eine Tendenz zur Psychologisierung. Der Krieg als „Erlebnis“, nicht als politischer, militärischer oder diplomatischer Vorgang, stand im Mittelpunkt der Besinnung. ${ }^{16}$ In Bäumers Texten ist das weibliche Mitgefühl tonangebend: Mit „unsere Generation, die deutschen Frauen“, oder einfach „wir“, spricht sie die deutsche Kollektive an. „Aber die unermeßlichen Opfer?", fragt die verantwortungsvolle Autorin. „Wie kommen die Frauen über die Grausamkeit dieser Tatsache hinweg?“ Die Frau sei dann zur „Hüterin und Pflegerin des Lebens" geworden, viele hätten nach religiösen Aufgaben gesucht, denn in allen Frauen sei „eine heilige Kraft““.17

Bäumer sprach auch über die Sinnlosigkeit des Todes, laut ihrer Metapher entkleide der Krieg den Tod seiner Sinnlosigkeit. Sie argumentiert gegen den bürgerlichen Tod, da man dort für nichts sterben könne. Im Gegensatz dazu stehe der Soldatentod, der "für“ etwas geschehe, ,eingefügt in die große Kette menschlichen Strebens und Ringens", was offenbar für das bürgerliche Leben und Sterben nicht gelten soll. ${ }^{18}$

Nach dem Lob des Soldatentodes legt Bäumer den Akzent auf die

12 Bäumer 1914: 6.

13 Bäumer 1914: 6.

14 Bäumer 1914: 6

15 Bäumer 1914: 30.

16 Flasch 2000: 293.

17 Bäumer 1914: 7.

18 Bäumer 1914: 10. 
weibliche Weltsicht. Dulce et decorum est pro patria mori, das „können die Frauen in tiefster Seele nachfühlen. Es ist ein mütterliches Grunderlebnis, daß Leben und Kraft hingeopfert werden muß, damit neues Leben um so schöner erblühen kann."19 Die deutschen Mütter, die ihren Sohn verloren haben, trösteten sich mit der „Größe des Schicksals, für das sie ausersehen waren“. Sie schließt mit einem ins Nationalistische gewendeten Paulus-Zitat: Den deutschen Frauen sei der „Tod verschlungen in den Sieg“. Im Neuen Testament bezog sich das auf die Auferstehung Christi, aber Bäumer erklärt, was der Satz für die deutschen Frauen 1914 bedeutet: Der Tod ihrer Söhne sei verschlungen in den „Sieg ihrer Enkel und Urenkel.“20

Während des Weltkrieges meldete sich auch eine ganz andere weibliche Stimme: Anette Kolb war in Deutschlan als Dichterin, Essayistin und Übersetzerin d bekannt. Sie schrieb 1914 im ersten Band des Zeit-Echo ihre Briefe an einen Toten; 1915 verfasste sie in den Weißen Blättern ihren Vortrag Die Internationale Rundschau und der Krieg; 1916 schrieb sie den Briefroman 13 Briefe einer Deutsch-Frañösin.

Aus einer neutralen Position schreibt die deutsch-französische Dichterin Annette Kolb abgegrenzt und zurückgezogen. Ihr Halt erlaubt ihr die Freiheit, um aufzuschreien und nachzudenken. In ihrem Buch versucht sie Lügenmeldungen über Kriegsereignisse objektiv zu untersuchen. Auch ihr Vortrag Die Internationale Rundschau und der Krieg wird in dem Buch wiedergegeben. Die beiden Textsorten - Jenseitsbriefe und Vortragstext - erlauben es der Autorin, Reflexionen in eine narrative Umgebung zu setzen, also theoretische Passagen mit genauer, leicht ironischer Situationsschilderung zu verbinden.

Sie unterscheidet sich von anderen Kriegsautoren, indem sie in ihren Kriegsreden ihren Ausgang, ihre Redevorbereitung, die Reaktion der Presse und des Publikums beschreibt. Der Redetext tritt dadurch auf mehreren Ebenen auf, wird vielfach gespiegelt. Auch er hat den Charakter eines Appells, aber ihm fehlt der Gestus der moralistischen Selbststilisierung, der auffordert, über Leiden und Trauer ,hinwegzukommen“. Die Autorin zensiert nicht ihre Erfahrung der Zerrissenheit als Deutsch-Französin, sondern versteht sich als Kundschafterin. In dieser extremen Situation macht sie Wahrnehmungen, die denjenigen verschlossen bleiben, die sich aus ihrer eindeutigen nationalen Bestimmung verstehen. Sie ist anders als ihre Zuhörer und sie denkt anders als ihr Publikum. Deswegen spricht sie und besteht darauf, ihre Rede zu Ende zu führen, auch wenn der Saal tobt. Den Gestus,

19 Bäumer 1914: 10.

20 Bäumer 1914: 10. 
mit dem sie dieses Recht durchsetzte, hatte sie vorher bei einer Münchner Schauspielerin eingeübt. ${ }^{21}$

Wie Kurt Flasch feststellt, erfindet Annette Kolb die mythische Metapher von Philoktet für den Intellektuellen im Weltkrieg. Anfang des 20. Jahrhunderts war die Geschichte des Philoktet noch bekannt: Der griechischen Sage zufolge hat Herakles dem Philoktet seinen Bogen und Pfeile geschenkt. Doch Philoktet wurde auf der Fahrt nach Troja von einer Schlange gebissen, und die Wunde heilte nicht. Sie strömte einen so furchtbaren Gestank aus, dass die Griechen ihren besten Kämpfer auf einer Insel zurückließen, wo er unter entsetzlichen Qualen neun Jahre lebte. Ohne den Bogen des Herakles war Troja aber nicht zu erobern und so holte Odysseus den Leidenden schließlich doch in das griechische Belagerungsheer vor Troja. Philoktet tötete Paris und Troja fiel.22

Annette Kolb betrachtet Philoktet als Urbild des Nachdenkers und Betrachters des Krieges, und zwar wegen seiner Schmerzensschreie:

"Wie Philoktet mit der schwärenden Fußwunde jede Betrachtung, die er faßt, was immer er sagt oder vernimmt, mit seinem Schmerzensgeheul unterbricht, so wird ihm jeder Gedanke zerrissen, jeder Schlag seines Herzens durch das Bewußtsein dieses grauenhaften Krieges zerhämmert.“23

Gedanken, Gefühle, Reden werden durch den Krieg zerrissen, man kann die Wahrheit nicht aussagen, man soll nur die Schmerzen unterdrücken. Sie bleiben fiktiv und die dichterische Fiktion enthüllt ihren fiktiven Charakter. Trotz aller Versuche kann man diesen Krieg nicht denken, denn

„vom Tag an, wo das Sengen und Brennen und Schießen und Erstechen und Erwürgen und Bombenwerfen und Minenlegen anging, von dem Tag an, siehst du, bin ich eine Ausgestoßene; von einer solchen Welt bin ich geschieden, wie ein Idiot. Denn ich verstehe ja nicht. Wie ein Idiot erschrecke ich vor den Menschen und fürchte mich seitdem. “24

Bei den Autorinnen unterscheidet man zwischen solchen, die gruppenorientiert waren und an die Frauen gerichtet von Ernährung, Krankenpflege, Kindererziehung und sozialem Bereich geschrieben haben, und Autorinnen, die nicht als Frau für Frauen geschrieben haben. Annette Kolb hat ihren Briefroman nicht speziell für Frauen geschrieben, auch nicht als

21 Kolb 1917: 12.

22 Flasch 2000: 298.

23 Kolb 1917: 126

24 Kolb 1917: 11. 
Frau, sondern als individuelle Person in einer Ausnahmesituation, in ihrem Fall als Deutsch-Französin im Krieg zwischen beiden Völkern.

Die Autorin Lily Braun war am Beginn des Weltkrieges eine der führenden Personen in der sozialistischen Frauenbewegung. Lily Braun war bekannt als Sozialpolitikerin, Publizistin und Romanschriftstellerin, sie ist 1916 gestorben. Ihr bekanntester Text Die Frauen und der Krieg zeigt einerseits allgemeine, eher philosophische Erwägungen zu Krieg, Frieden und der Bestimmung der Frau, andererseits nähert er sich der Sozialpolitik an. Sie glaubt, dass die Männer angesichts der Kriegsleistungen der Frau mehr Respekt vor ihren Fähigkeiten bekommen hätten, und betont dann die „Kulturbedeutung des Krieges“. ${ }^{25}$ Der Krieg habe gezeigt, dass der Staat die Frauen braucht. Sie schlägt auch für Frauen eine Dienstpflicht vor:

„Was würde der Staat besonders in schweren Zeiten ersparen und gewinnen, wenn er ein Heer von Frauen zur Verfügung hätte, von denen eine jede sofort wüßte, wo das kleine Rädchen ihrer Leistungskraft sich in die große Maschine einzufügen hat. Es gäbe keine Zankereien titelsüchtiger Weiber mehr und keine Fahnenflucht. Die Frauen würden endlich lernen, Uniform anzuziehen, in Reih und Glied zu marschieren, wenn es in die Schlacht geht." 26

Die Dichterin Lily Braun glaubte stark an den Staatssozialismus und plädierte gegen Frauen, die für eine geringe Kinderzahl verantwortlich waren. Ganz direkt rief sie die Frauen auf, „zum heiligen Gesetz ihrer Natur zurückzukommen durch den starken, bewußten Willen zur Mutterschaft““.27 Sie betrachtet es positiv, dass der Krieg den Intellektualismus und den Individualismus hinwegfege und der ,natürliche weibliche Instinkt” jetzt mit „elementarer Wucht zum Durchbruch" komme - gemeint ist die soziale Bestimmung der Frau. Der Text von Lily Braun macht auch auf die Vielfalt der Positionen innerhalb der deutschen Frauenbewegung aufmerksam.28

Die große Patriarchin Hedwig Dom veröffentlichte Das Land Goethes 1914/1916. Sie hatte schon 1873 das Frauenstimmrecht gefordert, wirkte als eine der größten Vertreterinnen der Frauenbewegung, und war fünfundachtzig Jahre alt, als sie schrieb:

„Wäre ich ein glühender Patriot - es zu sein heischt der Geist der Zeit - in meiner Seele würden Psalter und Harfe erwachen, und ich sänge dem Heroentum

\footnotetext{
25 Braun 1915: 48.

26 Braun 1915: 48.

27 Braun 1915: 53.

28 Flasch 2000: 304.
} 
des deutschen Volkes ein hohes Lied. Wäre ich ein Held der Feder - ich sage nicht Zeitungsschreiber - welchen Ranges und welcher Partei auch immer, von Heiligkeiten würden meine Kriegsbetrachtungen strahlend strotzen. Heilig, heilig der Krieg! heilig die Schlacht! heilig die Seligkeit des Sterbens auf dem Feld der Ehre, heilig der Feindeshaß! Gottes Donner hörte ich aus den Kanonen brüllen, und anstatt der dunklen Taube, der bombenwerfenden, Menschenleiber zerreißenden, sähe ich nur die weiße Taube, das Symbol der Ausgießung eines heiligen Geistes. Nach der Taube mit dem Ölzweig spähe ich vergebens aus.

Wäre ich ein Fatalist, ich spräche: es ist die Bestimmung der Völker, sich von Zeit zu Zeit gegenseitig zu vernichten. Wäre ich ein Teufel, ich täte lachen, lachen, in wollüstiger Schadenfreudejauchzend über den Sieg, den die Hölle errungen. Wäre ich der liebe Gott, ich beauftragte Petrus, meinen Pförtner, allen Kriegsanstiftern, Kriegshetzern, Kriegsbrünstigen und Kriegsliebenden die Himmelspforte zu sperren, mit dem Donnerwort: Unbefugten ist der Eingang nicht gestattet. Bin ich ein Mensch - nichts als ein Mensch - ich müßte ob dieses Krieges weinen, weinen, bis meine Augen vor Tränen blind geworden. Hedwig Dohm. “ 29

Hedwig Dohm war immer klar, wovon sie sich abgrenzte. Alle Haltungen, die sie selbst nicht teilen wollte, wie der Nationalismus, die Theologisierung des Krieges, der Fatalismus, die Kriegsbrunst und der Preis des Heldentodes, waren in demselben Buch doch vertreten. Die geistige Führerin der Frauenbewegung, die 1917 noch den Anti-Kriegstext Der Mißbrauch des Todes veröffentlichte, spricht hier auch nicht als Frau. Die einzige Rolle, die sie annimmt, ist die des Menschen. Sie muss Worte machen, um sich von herrschenden Kriegsphilosophien und ihrer Todesmystik abzusetzen. Sie muss echte Kriegstexte verfassen. Und zwar nicht als Frau, sondern als Mensch.

\section{Literatur}

\section{BÄUMER, Gertrud}

1914 Der Krieg und die Frau. Der deutsche Krieg. (Politische Flugschriften. Heft 15. Hg. von Ernst Jäckh) Stuttgart/Berlin: Deutsche Verlags-Anstalt

BRAUN, Lily

1915 Die Frauen und der Krieg. (Zwischen Krieg und Frieden. Heft 17.) Leipzig: Hirzel

29 Dohm 1916: 27. 
DOHM, Hedvig

1916 Das Land Goethes: 1914-1916: ein vaterländisches Gedenkbuch. In Isidor Landaur (Hg.): Berliner Goethebund. Stuttgart: DVA

FLASCH, Kurt

2000 Die geistige Mobilmachung. Die deutschen Intellektuellen und der Erste Weltkrieg. Ein Versuch. Berlin: Alexander Fest Verlag

$\mathrm{KOCH}$, Lars

2005 Der Erste Weltkrieg als Medium der Gegenmoderne: zu den Werken von Walter Flex und Ernst Jünger. (Epistemata - Würzburger wissenschaftliche Schriften. Reihe Literaturwissenschaft) Würzburg: Königshausen \& Neumann

2014 Der Erste Weltkrieg als kulturelle Katharsis und literarisches Ereignis. In Niels Werber - Stefan Kaufmann - Lars Koch (Hg.): Erster Weltkrieg. Kulturwissenschaftliches Handbuch. 97-141.

KOLB, Anette Stuttgart: Metzler

1917 Briefe einer Deutsch-Französin. 3. Aufl. Berlin: Verlag Erich Reiss MAYER, Matthias

2010 Der Erste Weltkrieg und die literarische Ethik. Historische und systematische Perspektiven. (Ethik - Text - Kultur) Paderborn: Wilhelm Fink Verlag 